\title{
Control of pain and dyspnea in patients with oncologic disease in acute care: non-pharmacological interventions
}

\begin{abstract}
Objective: To identify non-pharmacological strategies in the control of pain and dyspnea, in patient with oncological disease, in acute care.

Methodology: Question in PI[C]O format was used and search at EBSCO (MEDLINE with Full TEXT; CINAHL, Plus with Full Text; British Nursing Index) retrospectively from 2009 to 2015. We included also guidelines by reference entities: Oncology Nursing Society (2011) National Comprehensive Cancer Network and Cancer Care Ontario, resulting in a total of 15 articles.

Results: The gold standard for an adequate symptom control is a systematized assessment. Non-pharmacological measures: psycho-emotional support, hypnosis, counseling/training/instruction, therapeutic adherence, music therapy, massage, relaxation techniques, telephone support, functional and respiratory re-education increase health gains.
\end{abstract}

Conclusion: The control of oncologic pain and dyspnea require a comprehensive and multimodal approach

Keywords: oncologic pain, dyspnea, nursing interventions, acute care, music therapy, symptom, British nursing
Volume 2 Issue 3 - 2017

\author{
Ana Ramos, ' Ana Patrícia Tavares, ${ }^{2}$ Manuel \\ Lopes, ${ }^{2}$ Felismina Mendes, ${ }^{2}$ Pedro Parreira, ${ }^{3}$ \\ César Fonseca² \\ 'Hospital of Medium Tejo, Portugal \\ ${ }^{2}$ Department of Nursing, Univerisity of Coimbra, Portugal \\ ${ }^{3}$ Department of Nursing, University of Évora, Portugal
} Correspondence: César Fonseca, Department of Nursing,
Univerisity of Coimbra, Portugal,

Email cesar.j.fonseca@gmail.com

Received: January 12, 2017 | Published: February 13, 2017

\section{Introduction}

Globally, more than 14 million new cases of cancer will occur each year and the number of people with this disease expected to triple by 2030 , as a result of survival. ${ }^{1,2}$ Survivors continue to experience significant limitations, compared to those without a history of cancer. ${ }^{3}$ The presence of symptoms persists permanently, resulting from the direct adverse effects of cancer, treatment, exacerbation and/or onset of new ones, associated with recurrence or second tumor. ${ }^{4,5}$

Pain, dyspnea, fatigue, emotional distress arises simultaneously and is interdependent. In this way, the term cluster symptoms comes to light when two or more symptoms are interrelated, since they can share the same etiology and produce a cumulative effect on the person's functioning. ${ }^{6}$ Richards et al. ${ }^{7}$ found that patients with a high prevalence of pain were more likely to be treated with highdose pain relief than those who did not. The incidence of pain at the onset of the disease pathway is estimated at $50 \%$ and is increased to approximately $75 \%$ at advanced stages, which means that the survivor does not experience it only as an immediate treatment outcome. ${ }^{6}$ In an advanced stage of the ontological disease, dyspnea is one of the symptoms that assumes particular relevance, often arises associated with pain (about 45\%), represented a cluster symptoms inducer of greater anxiety and fatigue. That is responsible for the demand for health care, so it is fundamental a serious investment in their control. The objective of this systematic review of the literature is to identify non-pharmacological strategies in the control of pain and dyspnea, of the patients with oncological disease, in acute care.

\section{Research strategy}

We formulated a question in PI[C]O format: What are the nonpharmacological strategies (Intervention) for the control of pain and dyspnea (Outcomes) in the patients with oncology (Population) in acute care (Setting)? The electronic database used was based on EBSCO (MEDLINE with Full TEXT; CINAHL, Plus with Full Text, British Nursing Index), and descriptors were searched in the following order: [guideline OR guideline OR evidence based practice OR randomized controlled trial] AND [symptoms control OR dyspnea OR tachypnea OR Cheyne-stokes respiration OR respiratory symptoms OR chronic pain OR cancer pain OR [oncology nursing OR emergency care OR palliative care]. The descriptors were searched, retrospectively from 2009 until 2015, resulting in a total of 12 articles. Inclusion criteria include the guidelines emitted by reference entities in the Oncology Nursing Society, National Comprehensive Cancer Network ${ }^{9,10}$ and Cancer Care Ontario. Exclusion criteria included all articles with unclear methodology, repeated in both databases $(\mathrm{N}=3)$, aged less than 18 years and with a date prior to 2009. In total a total of 15 articles. As show the Figure 1. To assure its applicability in the clinical context, only levels of evidence considered of high quality, that is, up to $2 \mathrm{a}$, like shows the Table 1 , were acceptable. 


\section{Results}

Table I Review results.

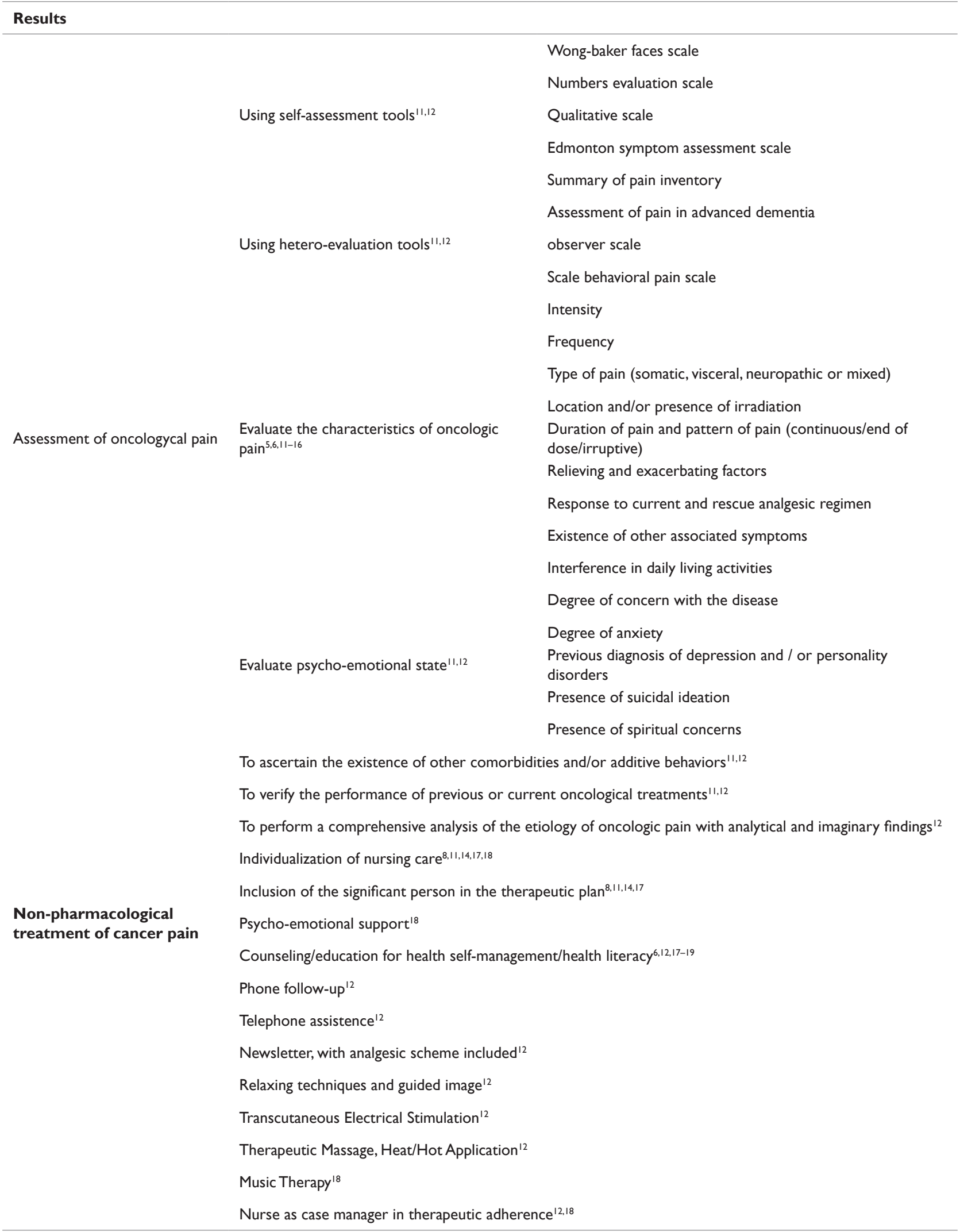


Table Continued...

Results

Onset: Beginning, frequency and duration

Provoking/Palliating: factors of relief and exacerbation

Quality description

Use the acronym O,P,Q,R,S,T,U e VIO

Region/Radiation: association with other symptoms

Severity: intensity

Treatment: therapeutic regimen, efficacy and adverse effects

Appreciation of Dyspnea Understanding: Understanding the etiology attributed

Values: objective in control of dyspnea

Evaluate the psycho-emotional state

To evaluate the existence of other comorbidities

Using assessment tools that include dyspnea: Edmonton Symptom Assessment Scale, Clinical Anxiety and Depression Scale - HADS, Modified Dyspnea Index (MDI)'

Screening of the sub treated causes of dyspnea, requiring pericardiocentesis, pelurodesis, thoracentesis, bronchofibroscopy, transfusion support or antibiotic therapy ${ }^{20}$

Control and Dissociation of Respiratory Times ${ }^{8,20}$

Effective assisted cough training 8,20

Positioning to reduce respiratory work ${ }^{8,20}$

Application of facial cold to stimulate the trigeminal nerve ${ }^{1,20}$

Consider the need to adopt healthy lifestyles ${ }^{8}$

Psycho-emotional support ${ }^{1,8,20}$

Management of anxiety of the person/caregiver/family, with exploration of the meaning of dyspnea for the person, illness and life expectancy ${ }^{1,8,20}$

Relaxing and visualization exercises ${ }^{1,8,20,21}$

Consider adjustment of nutritional and water habits ${ }^{8}$

Education for self-management of the therapeutic regimen ${ }^{8}$

Referral to other health services/professionals: pain unit, functional and respiratory rehabilitation, palliative care, mental and psychiatric health ${ }^{1,8,21}$

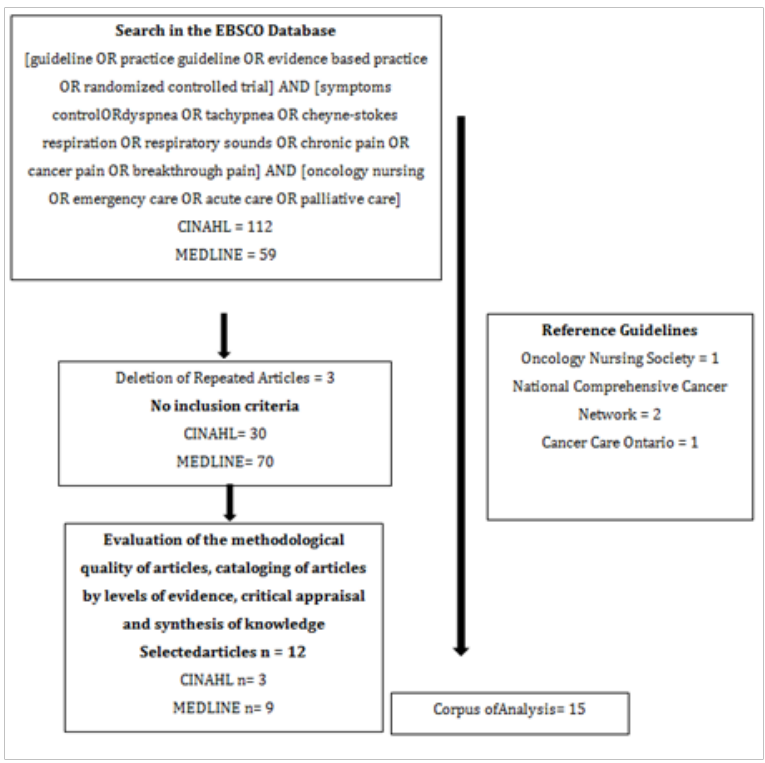

Figure I Research and selection process of articles, from 2009 to 2015.

\section{Discussion}

The assessment of pain is considered the first step towards effective pain control, which includes self-assessment tools that enable a more measurable dimension, where the person's speech is the gold standard in data collection. The characteristics of pain are influenced by the psycho-emotional state, the activities of daily living, and the existence of other comorbidities and/or additive behaviors. The performances of previous or current oncological treatments, analytical and imaging data were related to the etiology of pain. This aspect is considered fundamental in a comprehensive analysis of the person with cancer pain. ${ }^{10,22-24}$ The non-pharmacological strategies are the person-centered care, which emphasizes the individualization and inclusion of a significant person that increases health outcomes. Directed interventions for counseling, education for self-management, training/instruction, telephone follow-up, health literacy and nurses as case manager, with interconnection with other health professionals and health services increase therapeutic adherence and satisfaction with care. ${ }^{18,22,24,25}$ Therapeutic massage, hot and/or cold application, positioning, hypnosis, transcutaneous electrical nerve stimulation and music therapy are considered measures that improve the affectivity of medication regimen. ${ }^{25}$ 
In the evaluation of dyspnea, the literature suggests the use of the acronym $\mathrm{O}, \mathrm{P}, \mathrm{Q}, \mathrm{R}, \mathrm{S}, \mathrm{T}, \mathrm{U}$ and $\mathrm{V}$ to better evaluate its characteristics: (onset) frequency, provoking factors of relief and exacerbation, (Quality) description of the dyspnea sensation, (region/radiation) the existence of other symptoms simultaneously, (severity) intensity of dyspnea, (Treatment) medication used for its control, efficacy and adverse effects, (Understanding) dyspnea on human living, (Values) the level of acceptable/desirable dyspnea intensity for the person. The instruments to be included in its assessment are: the Edmonton System Assessment Scale, Clinical Anxiety and Depression Scale - HADS, Modified Dyspnea Index (MDI) and Numerical Rating Scale (NRS) for breathlessness, Modified Borg and Chronic Respiratory Questionnaire. ${ }^{26}$ The etiology of dyspnea should be carefully investigated to determine the need for other complementary techniques for its relief.

Non-pharmacological strategies aimed at functional and respiratory rehabilitation, cold application, adoption of healthy lifestyles, education for self-management /counseling, psychoemotional support and relaxation/ visualization exercises in anxiety control and referral to others health professionals/ services allow better control of dyspnea..$^{27,28}$

\section{Conclusion}

Pain is experienced by most cancer patients, and the incidence tends to increase in the more advanced stages of the disease. Dyspnea also appears as the main symptom, but arises mainly in the more advanced phases of the disease and frequently associated with pain. With regard to the control of pain and dyspnea, its assessment is paramount and fundamental through the use of appropriate scales. Non-pharmacological techniques should be person-centered in order to individualize the nurse's intervention. Its use in conjunction with pharmacological measures increases the effectiveness of the treatments bringing benefits to the patient in terms of pain and dyspnea control, but also proves to be beneficial in the control of anxiety and fatigue. Teamwork among health professionals allows better control of symptoms, increases adherence to the therapeutic regimen and satisfaction with health care.

\section{Acknowledgements}

None.

\section{Conflict of interest}

The authors declare no conflict of interest.

\section{References}

1. Ferlay J, Soerjomataram I, Ervik M, et al. Cancer incidence and mortality worldwide: sources, methods and major patterns in GLOBOCAN 2012 Int J Cancer. 2013;136(5):E359-358.

2. WHO. Global battle against cancer won't be won with treatment alone-effective prevention measures urgently needed to prevent cancer crises. Cent Eur J Public Health. 2014;22(1):23-28.

3. Zucca A, Boyes A, Linden W, et al. All's well that ends well? Quality of life and physical symptoms clusters in long-term cancer survivors across cancer types. J Pain Symptom Manage. 2012;43(4):720-731.

4. Sun V, Borneman T, Piper B, et al. Barriers to pain assessment and management in cancer survivorship. J Cancer Suviv. 2008;2(1):65-71.

5. Brant J, Beck S, Dudley W, et al. Symptom trajectories in post treatment cancer survivors. Cancer Nurs. 2011;31(1):67-77.
6. Cleeland C, Sloan J. Assessing the symptoms of cancer using patientreported outcomes (ASCPRO): searching for standards. J Pain Symptom Manage. 2010;39(6):1077-1085.

7. Richards C, Gisondi M, Chang C, et al. Palliative care symptom assessment for patients with cancer in the emergency department validation of the screen for palliative and end-of-life care needs in the emergency department instrument. J Palliat Med. 2011;14(6):757-764.

8. Dong S, Butow P, Costa D, et al. Symptom clusters in patients with advanced cancer: a systematic review of observational studies. $J$ Pain Symptom Manage. 2014;48(3):411-450.

9. National comprehensive cancer network. NCCN guidelines palliative care. Washington: National Comprehensive Cancer Network. 11;2011.

10. National comprehensive cancer network. NCCN clinical practice guidelines in oncology: adult cancer pain. Washington: National Comprehensive Cancer Network; 2014.

11. Farquhar M, Prevost A, McCrone P, et al. Study Protocol: Phase III single-blinded fast-track pragmatic randomised controlled trial of a complex intervention for breathlessness in advanced disease. Trials. 2011;12(130):1-11.

12. Heidenreich A, Bastian P, Bellmunt J, et al. Guidelines on prostate cancer. Netherlands: European Association of Urology; 2013.

13. Bharkta H, Marco C. Pain management: association with patient satisfaction among emergency department patients. J Emerg Med. 2014;46(4):456-464.

14. Caraceni A, Hanks G, Kaasa S, et al. Use of opioid analgesic in the treatment of cancer pain: evidence-based recommendations from the EAPC. Lancet Oncol. 2012;13(2):e58-68.

15. Choi M, Kim H, Chung S, Ahn M, Yoo J, et al. Evidence-based practice for pain management for cancer patients in an acute care setting. Int $J$ Nurs Pract. 2014;20(1):60-69.

16. LeBlanc T, Abernethy A. Building the palliative care evidence base: lessons from a randomized controlled trial of oxygen vs. room air for refractory dyspnea. J Natl ComprCancNetw. 2014;12(7):989-992.

17. Dale O, Moksnes K, Kaasa S. European Palliative Care Research Collaborative pain guidelines: opioid switching to improve analgesia or reduce side effects: a systematic review. Palliat Med. 2010;25(5):494503.

18. Jarzyana D, Jungquist C, Pasero C, et al. American society for pain management nursing guidelines on monitoring for opioid-induced sedation and respiratory depression. Pain Manag Nurs. 2011;12(3):118145 .

19. Doran D. Nursing-sensitive outcomes: state of the science. USA: Jones and Bartlett; 2003.

20. Fielding F, Sanford T, Davis M. Achieving effective control in cancer pain: a review of current guidelines. Int J Palliat Nurs. 2013;19(12):584 591.

21. Guyatt G, Oxman A, Visit G, et al. GRADE: an emerging consensus on rating quality of evidence and stregth of recommendations. $B M J$. 2008;336(7650):924-926.

22. Ripamonti C, Santini D, Maranzo E, et al. Management of cancer pain: ESMO clinical practice guidelines. Ann Oncol. 2012;23:139-154.

23. Yamaguchi T, Shima Y, Morita $T$, et al. Clinical guidelines for pharmacological management of cancer pain: the Japanese Society of Palliative Medicine recommendations. Jpn JClin Oncol. 2013;43(9):896909 .

24. Wengström Y, Geerling J, Rustoen T. European oncology nursing society breakthrough cancer pain guidelines. Eur J Oncol Nurs. 2014;18:127131. 
25. Vallerand A, Musto S, Polomano R. Nursing's role in cancer pain management. Curr Pain Headache Rep. 2011;15:250-262.

26. Raymond V, Bak K, Kiteley C, et al. Symptom management guide-topractice: dyspnea. Canada: Cancer Care Ontario; 2010.

27. DiSalvo WM, Joyce MM, Tyson LB, et al. Putting evidence into practice: evidence-based interventions for cancer-related dyspnea. Clin J Oncol Nurs. 2012;12(2):341-352.
28. Zeppetella G. Opioids for the management of breakthrough cancer pain in adults: A systematic review undertaken as part of an EPCRC opioid guidelines project. Palliat Med. 2010;25(5):516-524. 